Research Article

\title{
Terahertz Absorption Characteristics of the Sodium Carboxymethyl Cellulose Colloid Based on Microfluidic Technology
}

\author{
Chenxin Ding $\mathbb{C}^{1},{ }^{1}$ Bo Su $\mathbb{C}^{1,},{ }^{1,2,3,4}$ Guoyang Wang $\mathbb{C}^{1,2,3,4}$ Qinghao Meng $\mathbb{C}^{1,2,3,4}$ \\ Jiahui Wang $\mathbb{D}^{1,2,3,4}$ and Cunlin Zhang $\mathbb{D}^{1,2,3,4}$ \\ ${ }^{1}$ Department of Physics, Capital Normal University, Beijing 100048, China \\ ${ }^{2}$ Beijing Advanced Innovation Centre for Imaging Theory and Technology, Beijing 100048, China \\ ${ }^{3}$ Beijing Key Laboratory for Terahertz Spectroscopy and Imaging, Beijing 100048, China \\ ${ }^{4}$ Key Laboratory of Terahertz Optoelectronics, Ministry of Education, Beijing 100048, China
}

Correspondence should be addressed to Bo Su; subo75@cnu.edu.cn

Received 18 February 2021; Revised 12 April 2021; Accepted 25 August 2021; Published 6 September 2021

Academic Editor: E. Bernabeu

Copyright ( $\odot 2021$ Chenxin Ding et al. This is an open access article distributed under the Creative Commons Attribution License, which permits unrestricted use, distribution, and reproduction in any medium, provided the original work is properly cited.

Sodium carboxymethyl cellulose is a type of macromolecular chemical substance that is widely used in the industry for food thickening. In this study, terahertz and microfluidic technologies were combined, and a microfluidic chip with a channel depth of $50 \mu \mathrm{m}$ was fabricated to carry samples. The terahertz characteristics of the sodium carboxymethyl cellulose colloid were studied at different concentrations and applied electric fields. The obtained results showed that different concentrations of sodium carboxymethyl cellulose have different time-domain spectra; with an increase in concentration, the terahertz transmittance of sodium carboxymethyl cellulose decreased. Under the applied electric field treatment, the longer the electric field acting time is, the higher the terahertz transmission intensity is. This approach is a safe and reliable new method for the determination of sodium carboxymethyl cellulose concentration, which provides technical support for the in-depth study of sodium carboxymethyl cellulose.

\section{Introduction}

Terahertz $(\mathrm{THz})$ is the electromagnetic radiation in the frequency range of $0.1-10 \mathrm{THz}$ and the wavelength range of $30 \mu \mathrm{m}-3 \mathrm{~mm}$. The $\mathrm{THz}$ region is between the infrared and microwave regions, and it has not been fully exploited. One advantage of $\mathrm{THz}$ technology arises from the low-energy photons of $\mathrm{THz}$ waves, which range from less than $1 \mathrm{meV}$ to a few tens of $\mathrm{meV}$ [1]. Because the $\mathrm{THz}$ wavelength is long and the energy is low, it does not ionize the sample and can be used for nondestructive testing of materials; thus, the use of $\mathrm{THz}$ waves for material testing has the advantages of safety and reliability. The characteristic vibration modes of many chemical reagents and biological macromolecules (such as proteins or DNA) and sugars happen to be in the $\mathrm{THz}$ range [1-7]. This characteristic provides a reliable basis for the study of some macromolecules using $\mathrm{THz}$ technology. With the development of $\mathrm{THz}$ technology, its application field and scope are wider. Wu et al. [2] studied the characteristics of four electrolyte solutions (i.e., $\mathrm{KCl}, \mathrm{KBr}$, $\mathrm{MgCl}_{2}$, and $\mathrm{CaCl}_{2}$ ) in the range of $0.1-1.0 \mathrm{THz}$ using $\mathrm{THz}$ technology. It is determined that different electrolytes can cause association and destruction of hydrogen bonds in water, which affects the $\mathrm{THz}$ absorption of water. Nishizawa et al. [3] measured the $\mathrm{THz}$ absorption spectra of 20 amino acids. Yu et al. [4] studied the temperature characteristics of four amino acids at room temperature and 250, 200, 150, $100,70,40,10$, and $4.5 \mathrm{~K}$. The obtained results showed that the response of the $\mathrm{THz}$ spectra of aliphatic and aromatic amino acids to temperature was different. Fan et al. [5] studied different concentrations of potassium chloride and potassium iodide solutions and determined that the $\mathrm{THz}$ 
transmittance of potassium chloride solution decreased with an increase in concentration, whereas that of potassium iodide solution increased. Currently, some progress has been made in using $\mathrm{THz}$ technology to detect differences in the molecular structure, and quantitative studies have been made on the properties of different chemical reagents varying with temperature. However, the exploration of concentration is limited to solutions, and there are fewer studies on colloids.

In production and life, many substances are liquid. Most detection methods of liquid samples use a labelling method or introduce another reagent; however, these methods have a major flaw; that is, they cause irreversible damage and contamination to the samples. Additionally, it is relatively difficult to study the $\mathrm{THz}$ transmission spectrum of liquid samples owing to the strong absorption of $\mathrm{THz}$ radiation by water. There are two main ways to solve this problem: either use high-power laser or reduce the amount of test samples. However, the cost of increasing the power is high, and the feasibility is low. Therefore, reducing the sample amount is the optimal solution. Microfluidic technology can make the depth of a microchannel reach the order of microns. Thus, the distance between $\mathrm{THz}$ wave and water becomes smaller, and the absorption of water to $\mathrm{THz}$ wave is reduced. $\mathrm{Si}$ multaneously, the practical sample amount is preserved [6]. Using quartz and polydimethylsiloxane (PDMS), Han et al. [7] developed a microfluidic chip to measure the $\mathrm{THz}$ transmittance of water. The obtained results showed that the absorption coefficient of water monotonically increases with an increase in frequency in the frequency range from 0.2 to $1 \mathrm{THz}$. Leclerc et al. [8] explored a direct and fast method to manufacture a microfluidic chip system using a polystyrene plate and computer micromachining technology. Alfihed et al. [9] used the $\mathrm{THz}$ technology to detect various copolymers (e.g., PDMS, polycarbonate, and polyethylene terephthalate) and obtained stable $\mathrm{THz}$ absorption coefficient of polyethylene at a certain $\mathrm{THz}$ frequency, which showed the viability of the development of microfluidic technology.

Sodium carboxymethyl cellulose (CMC-Na) can be used as an adhesive in industrial production, a thickening agent in food, and a flocculant in sewage treatment. Control of concentration is essential in preparation and use. In the past, electrochemistry, osmotic pressure, and other methods were used to detect colloidal concentration. These methods not only destroyed samples but also required a relatively long time for detection. In this study, microfluidic and $\mathrm{THz}$ technologies were combined to explore different concentrations of the CMC-Na colloid, and the feasibility of the method was confirmed. Additionally, the $\mathrm{THz}$ absorption characteristics of CMC-Na were tested under the applied electric field treatment.

\section{Experimental System and Devices}

2.1. Light Path System. This experimental system uses a photoconductive antenna to generate and detect $\mathrm{THz}$ waves. The core component of the entire system is a femtosecond fibre laser with a central wavelength of $1550 \mathrm{~nm}$, a pulse repetition frequency of $100 \mathrm{MHz}$, and an output power of $130 \mathrm{~mW}$. The laser beam is divided into two beams after passing through the half-wave plate and PBS crystal. A beam of pump light is coupled into a fibre-optic photoconductive antenna (BPCA-100-05-10-1550-C-F) through a mechanical translation platform to generate $\mathrm{THz}$ waves. The other beam, as detection light, is coupled into a fibre-optic photoconductive antenna (Batop BPCA-180-05-10-1550-C-F) to detect $\mathrm{THz}$ waves. Samples of different concentrations were injected into the microfluidic chip and placed vertically between two off-axis parabolic mirrors with a diameter of $50 \mathrm{~mm}$. The diameter of the $\mathrm{THz}$ spot on the microfluidic chip is $3 \mathrm{~mm}$. After passing through the microfluidic chip, the $\mathrm{THz}$ wave generated by the photoconductive antenna carries the sample information, which is then detected by the detection antenna. Finally, the signal is amplified by the phase-locked amplifier, and the data are collected and processed by using the computer. The experimental optical path system is shown in Figure 1.

2.2. Fabrication of the Microfluidic Chip. The materials used for microfluidic chips usually include quartz crystal, polyethylene, and cycloolefin copolymer (COC). Polyethylene is opaque to visible light, so it is not easy to observe the amount of liquid in the chip. The terahertz transmittance of the quartz crystal is $90 \%[10,11]$. However, COC has a stable $\mathrm{THz}$ absorption and a high transmittance of more than $96 \%$ with frequency variation [12], which makes it the best material for the microfluidic chip.

In this study, two COC plates $(4 \mathrm{~cm} \times 4 \mathrm{~cm} \times 0.2 \mathrm{~cm})$ were used as the substrate and cover of the microfluidic chip, and there were liquid inlet and outlet ports on the substrate and cover, respectively. A strong double-sided adhesive with a thickness of $50 \mu \mathrm{m}$ was used to bond the microfluidic chip. The middle of the double-sided adhesive tape is made into a hollow pattern according to the shape of the groove so that the hollow part in the bonding process forms the groove of the microfluidic chip, and the size of the groove is $3 \mathrm{~cm} \times 1 \mathrm{~cm} \times 50 \mu \mathrm{m}$. The fabrication of the microfluidic chip is shown in Figure 2.

2.3. Applied Electric Field Device. The schematic diagram of the applied electric field device used in this study is shown in Figure 3. A high-voltage power supply (DW-P153-0.5C51) is used to generate a uniform electric field through two metal plates parallel to each other. The electric field size is about $8270 \mathrm{~V} / \mathrm{cm}$. The microfluidic chip is placed between two metal plates for different times of electric field processing, and then the two metal plates are removed, and the $\mathrm{THz}$ TDS system is used for transmission detection of the microfluidic chip.

2.4. Preparation of the $\mathrm{CMC}-\mathrm{Na}$ Colloid. To eliminate the interference of other ions in water, this experiment used deionized water to prepare the CMC-Na colloid. The CMC$\mathrm{Na}$ powder was weighed with an electronic scale $(0.01 \mathrm{~g}$ accuracy), and the appropriate proportion of deionized water was measured in a measuring cylinder. After mixing in 


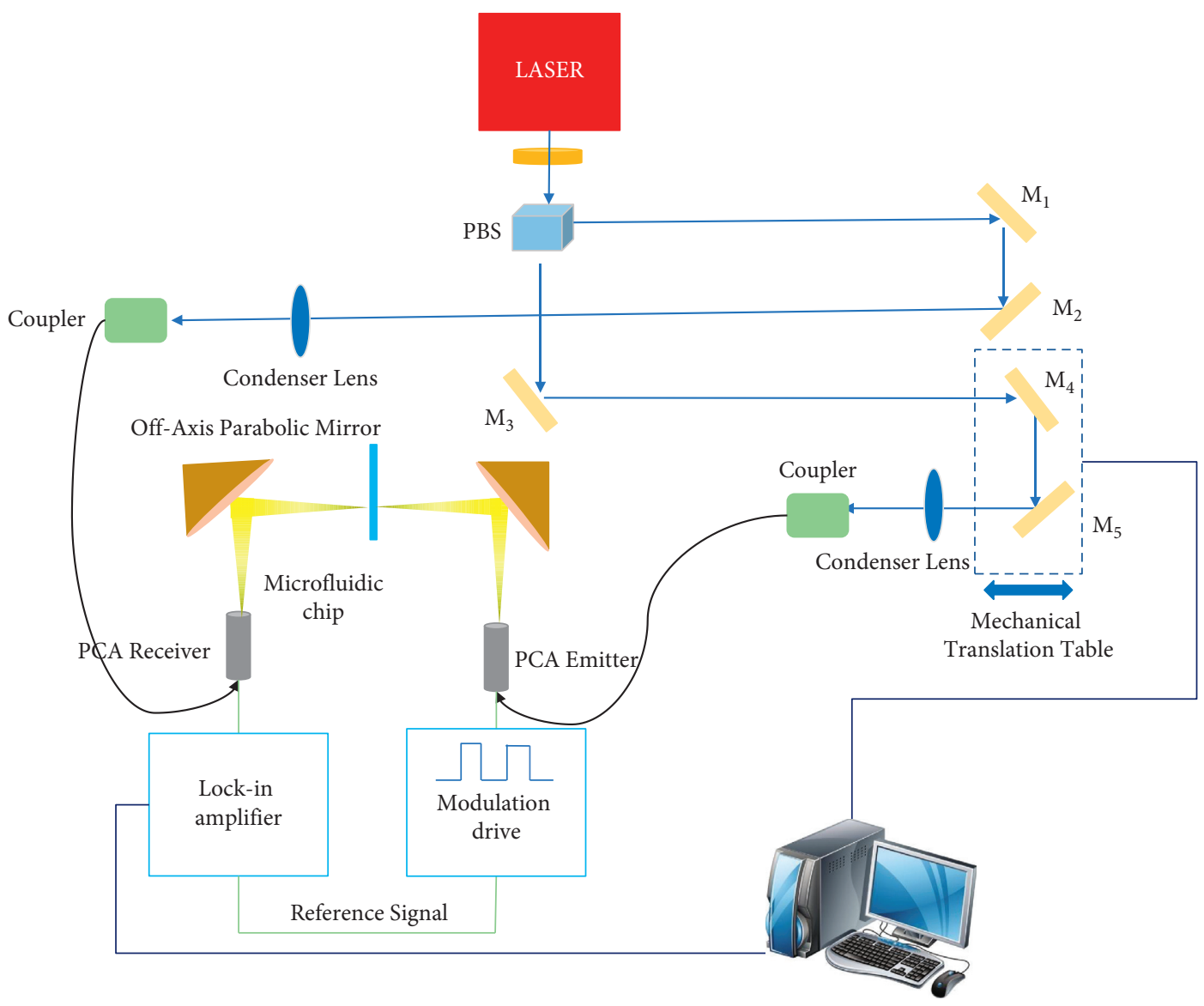

FIGURE 1: Experimental light path system diagram.

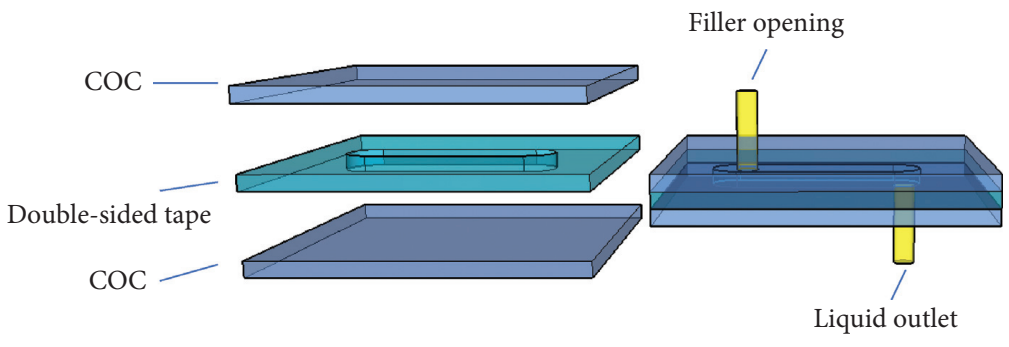

(a)

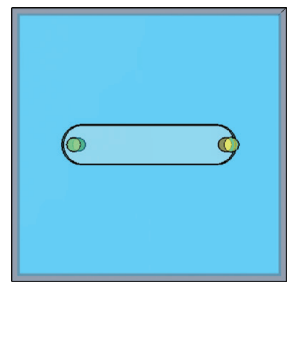

(b)

Figure 2: Schematic diagram of the microfluidic chip: (a) schematic diagram of the preparation process; (b) chip top view.

a beaker, heating in a water bath at $50^{\circ} \mathrm{C}$, and stirring with a glass rod, four types of the uniform CMC-Na colloid were prepared at concentrations of $0.5 \%, 1 \%, 1.5 \%$, and $2 \%$. Because CMC-Na has thermal stability below $80^{\circ} \mathrm{C}$, the water bath will not destroy the characteristics of CMC-Na but will make the preparation of the colloid faster.

\section{Experimental Procedures}

3.1. $\mathrm{THz}$ Transmission Characteristics of the CMC-Na Colloid at Different Concentrations. The CMC-Na colloid was injected into the microfluidic chip with a syringe through the liquid inlet and placed vertically in the middle of the off-axis parabolic mirror of the $\mathrm{THz}$ time-domain spectroscopy
(THz-TDS) system. To avoid differences caused by different microfluidic chips, in this experiment, the same microfluidic chip was used for the detection of colloid with different concentrations. After each detection was completed, another syringe was used to absorb distilled water and inject it through the liquid inlet to wash the microfluidic chip repeatedly. Air is then injected into the chip, distilled water is expelled, and the next sample to be tested is injected. The detection was carried out in the order of distilled water, $0.5 \%, 1 \%, 1.5 \%$, and $2 \%$ concentrations. The path of the microfluidic chip in the beam was removed and repositioned, and each concentration was measured three times. Each concentration of the colloid is reinjected into the microfluidic chip, and the above procedure is repeated. 


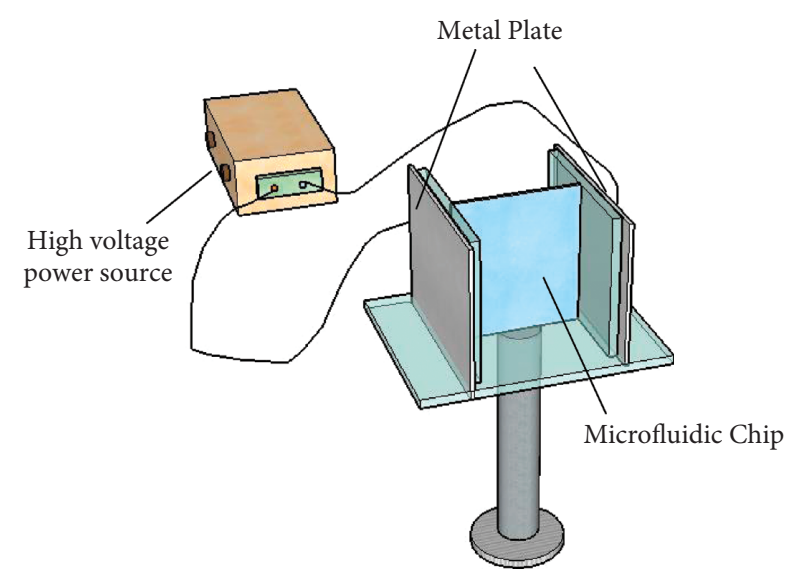

FIgURE 3: Schematic diagram of the applied electric field device.

Figure 4 shows the $\mathrm{THz}$ time-domain spectra of CMC-Na colloids with different concentrations. It can be seen that the amplitudes of the $\mathrm{THz}$ time domain decrease with the increase of $\mathrm{THz}$ concentration. The error of this study is analyzed by taking the $0.5 \%$ concentration colloid as an example. As shown in Figure 5, the difference between colloids is greater than the standard deviation of repeated measurements (repeatability). As shown in Figure 6, the difference between repeated injections of the same sample is greater than the standard deviation of repeated injections (reproducibility). All the samples of concentration in this study satisfied the above rules, which indicated the validity of the data.

\subsection{Effect of the Applied Electric Field on the THz Transmission} Characteristics of the CMC-Na Colloid. The 1\% CMC-Na colloid was injected into the microfluidic chip. Under the condition of continuous external electric field, after 10 minutes, the microfluidic chip was detected for the first time, and then after 20 minutes, the microfluidic chip was detected again. Figure 7 shows the time-domain spectra. It is determined that, with an increase in the applied electric field action time, the intensity of the time-domain spectra increases, which indicates that the addition of electric field changes the transmission characteristics of the $\mathrm{THz}$ wave.

\section{Results and Discussion}

Owing to the hydrogen bonds between water molecules, water strongly absorbs $\mathrm{THz}$ waves. The formation process of hydrogen bonds is as follows. A hydrogen atom is covalently bonded to the highly electronegative and small-radius atom $X$, and the electron cloud near the hydrogen atom is shifted toward the $X$ atom, which causes the hydrogen atom to appear almost as a proton. In this case, there are no inner electrons, and the hydrogen atom with a partial positive charge forms an electrostatic force with a negative electron cloud surrounding the $Y$ atom. This electrostatic force is called a hydrogen bond. There are many carboxyl groups $(-\mathrm{COOH})$ and hydroxyl groups $(-\mathrm{OH})$ in CMC-Na macromolecules [13], and carboxyl and hydroxyl groups can

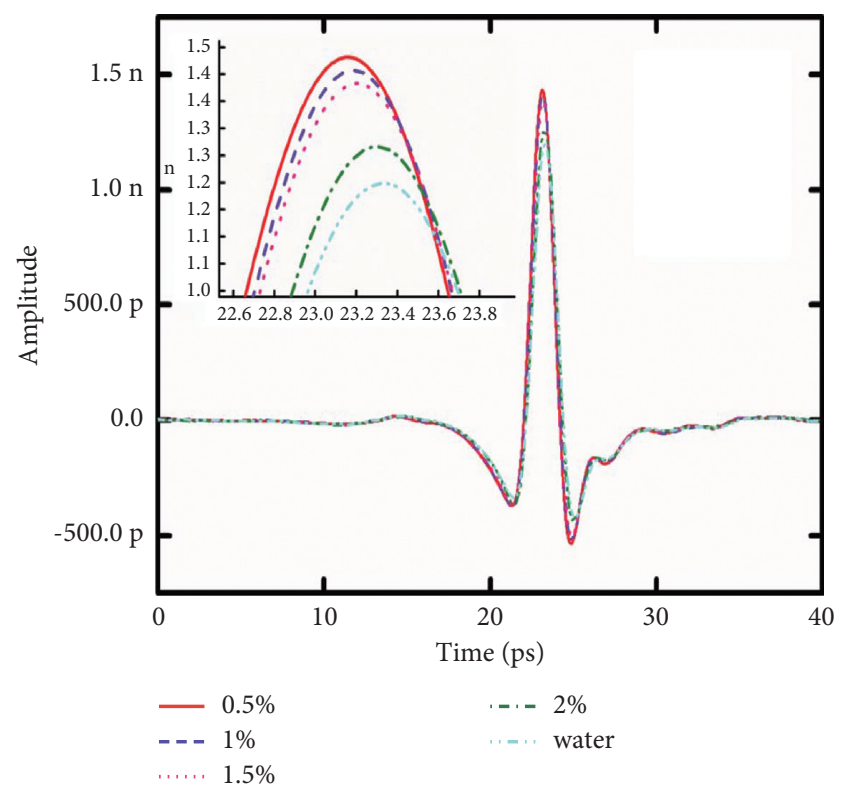

FIgURE 4: THz time-domain spectra of the CMC-Na colloid at different concentrations.

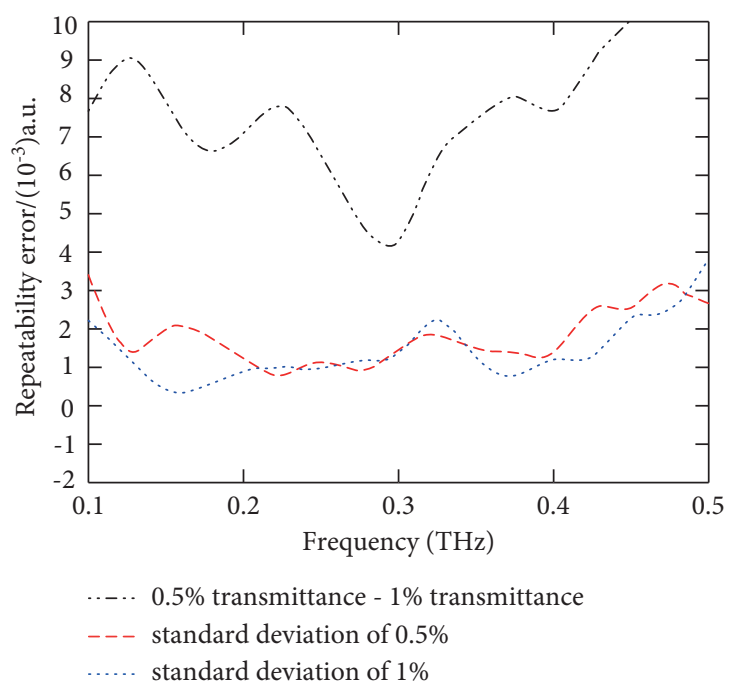

FIGURE 5: Repeatability error analysis.

form hydrogen bonds with water molecules [14]. A new hydrogen bond was formed between the CMC-Na macromolecule and the water molecule, which reduced hydrogen bonding between water molecules. In addition to carboxyl and hydroxyl groups, there is also $\mathrm{Na}^{+}$in the CMC-Na colloid. Wang et al. [15] studied the effect of ions on the structure of water, related the influence of ions on the structure of water to the viscosity coefficient, and analyzed the influence degree of different ions on the structure of water, among which $\mathrm{Na}^{+}$had little influence on the structure of water. Therefore, in this study, the influence of $\mathrm{Na}^{+}$can be excluded. Sun [14] studied the influence of hydrophobic and hydrophilic groups on the water structure by Raman spectroscopy, and the obtained results showed that adding hydrophilic groups affected hydrogen bonds in water. Wen 


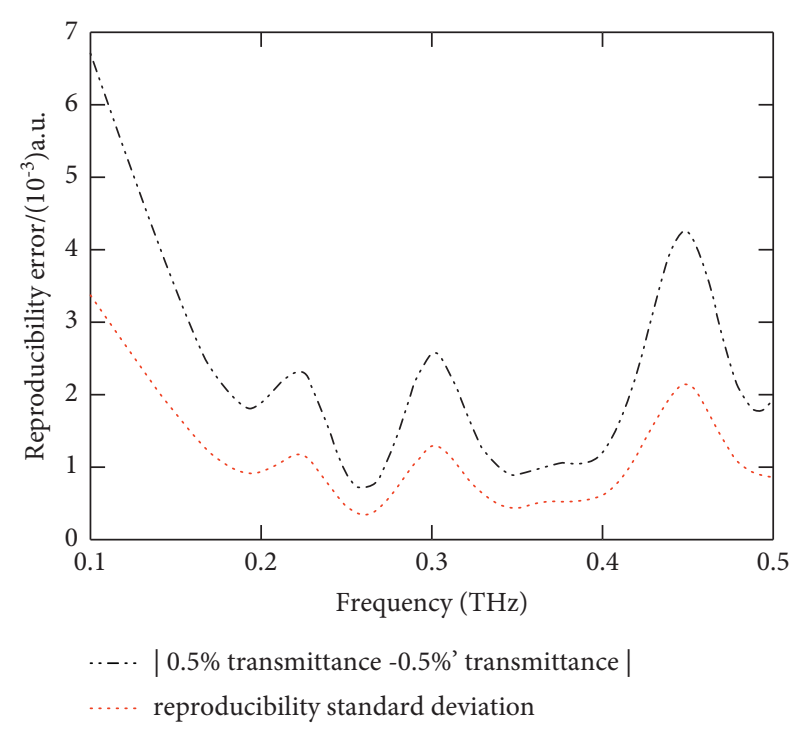

FIgURE 6: Error analysis of repeated injection of the same sample.

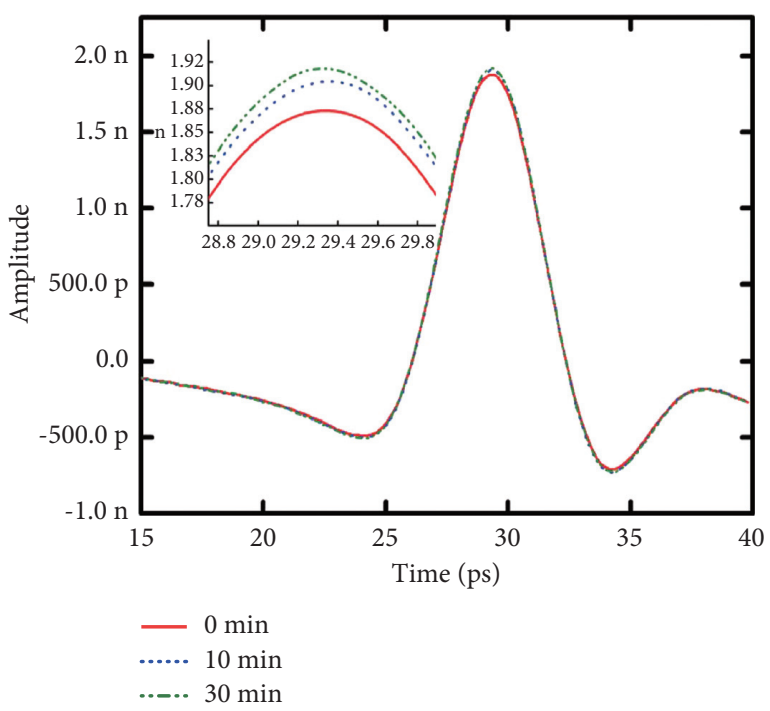

FIgURE 7: Time-domain spectra of the THz transmission intensity of the CMC-Na colloid located in an electric field for different times.

et al. [16] determined that a hydrogen bond could be formed between CMC-Na and silk fibroin protein, which reduced hydrogen bonding between silk fibroin protein molecules. Liu et al. [17] determined that, in the blending process of CMC-Na and wool keratin, owing to the interaction of the hydrogen bond, the intramolecular hydrogen bond force of keratin was reduced. Therefore, it can be preliminarily recognised that, with an increase in colloid concentration, the number of newly formed hydrogen bonds between CMC-Na molecules in a colloid and water molecules increases, which results in the variation of the $\mathrm{THz}$ transmission spectrum with concentration.

Several authors $[18,19]$ have calculated the refractive index and absorption coefficient of the sample using the THz-TDS system using the Kramers-Krönig relation:

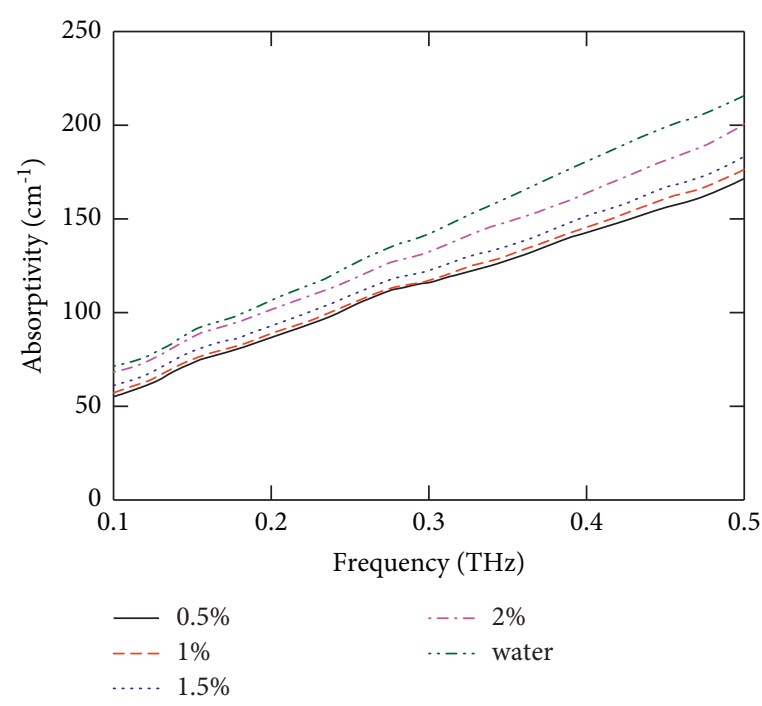

FIgURE 8: THz absorption coefficients of the CMC-Na colloid at different concentrations.

$$
\begin{gathered}
n_{1}(\omega)=\frac{c \varphi(\omega)}{\mathrm{d} \omega}+1, \\
n_{S}(\omega)=1-\frac{c \varphi(\omega)}{\mathrm{d} \omega}, \\
\alpha_{s}=\frac{2}{d} \ln \frac{n_{s}\left(n_{1}+1\right)^{2}}{\rho(\omega) \cdot\left(n_{1}+n_{s}\right)^{2}} .
\end{gathered}
$$

Equation (1) is used to calculate the refractive index of COC. Equation (2) is used to calculate the sample refractive index. Equation (3) is used to calculate the sample absorption coefficient. $n_{s}$ is the sample refractive index; $c$ is the speed of light; $\varphi(\omega)$ and $\rho(\omega)$ are the phase ratio and amplitude ratio of the sample and reference signal given by the THz-TDS system, respectively; $\alpha_{s}$ is the absorption coefficient of the sample; $n_{1}$ is the COC refractive index in this study; and $d$ is the sample thickness. The $\mathrm{THz}$ absorption coefficient of the CMC-Na colloid at different concentrations can be obtained using the aforementioned equation (3), as shown in Figure 8. The terahertz absorption coefficient of water in this study is basically consistent with that of Kindt and Schmuttenmaer [20] and Son et al. [21]. It can be seen that the absorption coefficient increases with an increase in colloid concentration, which is consistent with the result that the higher the colloid concentration, the lower the $\mathrm{THz}$ transmission in the aforementioned experiment.

In this study, the applied electric field has an effect on the $\mathrm{THz}$ transmission intensity. The longer the time of the applied electric field, the higher the $\mathrm{THz}$ transmission intensity of the CMC-Na colloid. The applied electric field can reduce hydrogen bonding and increase the diffusion coefficient of water molecules [22]. Therefore, it can be preliminarily concluded that the applied electric field reduces the effect of hydrogen bonding in the sample, and the $\mathrm{THz}$ absorption is weakened; thus, the $\mathrm{THz}$ transmission intensity increases with the increase of the applied electric field time. 


\section{Conclusions}

In this study, the $\mathrm{THz}$ absorption characteristics of the CMC$\mathrm{Na}$ colloid at different concentrations were explored by combining microfluidic technology with $\mathrm{THz}$ technology. Through the analysis of the quartz crystal, polyethylene, and COC, this study selected COC to make the microfluidic chip and detected the CMC-Na colloid of different concentrations. The obtained results show that the intensity of the $\mathrm{THz}$ timedomain spectrum decreases with an increase in the CMC-Na colloid concentration. It is preliminarily believed that CMC$\mathrm{Na}$ molecules in the colloid form hydrogen bonds with water molecules, which reduce hydrogen bonding between water molecules. The applied electric field breaks hydrogen bonds in the aqueous solution and weakens the vibration of polar molecules, which weakens $\mathrm{THz}$ absorption and enhances sample transmission. The material of the microfluidic chip in this study has high terahertz transmittance, and the preparation method of the microfluidic chip is simple, which has a wide application prospect in the study of liquid biological samples. The introduction of an external electric field improves the transmission intensity of the terahertz wave, provides a reference method for improving the detection sensitivity of terahertz technology, and plays a positive role in expanding the application range of terahertz technology.

\section{Data Availability}

The data used to support the findings of this study are available from the corresponding author upon request.

\section{Conflicts of Interest}

The authors declare that they have no conflicts of interest.

\section{Authors' Contributions}

All authors contributed to the theoretical analysis, calculations, experiment, and preparation of the manuscript.

\section{Acknowledgments}

This work was financially supported by the National Natural Science Foundation of China (NSFC) (61575131) and the Beijing Municipal Commission of Education (no. SQKM201810028004). The authors would like to thank 'Enago' for providing English touch up.

\section{References}

[1] C. L. Zhang, Terahertz Sensing and Imaging, 2008.

[2] Y. X. Wu, B. Su, J. S. He, and C. L. Zhang, "Terahertz Absorption Characteristics of electrolyte solution based on microfluidic chip," Spectroscopy and Spectral Analysis, vol. 39, no. 8, pp. 2348-2353, 2019.

[3] J. Nishizawa, T. Sasaki, K. Suto et al., "Frequency-tunable terahertz-wave generation from $\mathrm{GaP}$ using Cr:forsterite lasers," International Journal of Infrared and Millimeter Waves, vol. 27, no. 7, pp. 923-929, 2006.

[4] J. P. Yu, C. Li, W. T. Yi, and B. B. Jin, "Temperature dependence of terahertz spectra of amino acids,"
Spectroscopy and Spectral Analysis, vol. 38, no. 2, pp. 386-393, 2018.

[5] N. Fan, B. Su, Y. X. Wu, and H. F. Zhang, "Sandwich terahertz microfluidic chip," Spectroscopy and Spectral Analysis, vol. 38, no. 5, pp. 1362-1367, 2018.

[6] X. Ju, W. Yang, S. Gao, and Q. Li, "Direct writing of microfluidic three-dimensional photonic crystal structures for terahertz technology applications," ACS Applied Materials \& Interfaces, vol. 11, no. 44, pp. 41611-41616, 2019.

[7] X. Han, B. Su, and C. L. Zhang, "A terahertz microfluidic chip," Journal of Terahertz Science and Electronic Information Technology, vol. 13, no. 4, pp. 536-539, 2015.

[8] C. A. Leclerc, S. Williams, C. Powe et al., "Rapid design and prototyping of microfluidic chips via computer numerical control micromilling and anisotropic shrinking of stressed polystyrene sheets," Microfluidics and Nanofluidics, vol. 25, no. 2, 2021.

[9] S. Alfihed, M. H. Bergen, A. Ciocoiu, F. H. Jonathan, and G. F. Ian, "Characterization and integration of terahertz technology within microfluidic platforms," Micromachines, vol. 9, no. 9, 2018.

[10] R. Kitamura, L. Pilon, and M. Jonasz, "Optical constants of silica glass from extreme ultraviolet to far infrared at near room temperature," Applied Optics, vol. 46, no. 33, pp. 8118-8133, 2007.

[11] S. Tsuzuki, N. Kuzuu, H. Horikoshi, and S. Katsuya, "Influence of OH-group concentration on optical properties of silica glass in terahertz frequency region," Applied Physics Express, vol. 8, no. 7, Article ID 072402, 2015.

[12] A. Sengupta, A. Bandyopadhyay, B. F. Bowden, B. F. Bowden, J. A. Harrington, and J. F. Federici, "Characterisation of olefin copolymers using terahertz spectroscopy," Electronics Letters, vol. 42, no. 25, pp. 1477-1479, 2006.

[13] A. L. Wu and L. Q. Zheng, "Preparation and controlled release of sodium carboxymethyl cellulose gel carrier," China Cleaning Industry, vol. 2016, no. 2, 12 pages, Article ID 9676949, 2016.

[14] Q. Sun, "The effects of dissolved hydrophobic and hydrophilic groups on water structure," Journal of Solution Chemistry, vol. 12, 2020.

[15] W. H. Wang, L. Zhao, and B. Yan, "Effect of ions on water structure," Chemistry, vol. 6, pp. 491-498, 2010.

[16] D. J. Wen, H. Wang, X. S. Zhu, and K. Shigenori, "Study on mechanical properties of silk fibroin/carboxymethyl cellulose membrane," Journal of Textile Research, vol. 25, no. 4, 2004.

[17] S. P. Liu, L. Li, R. T. Liu, and Y. Wang, "Structure and properties of sodium carboxymethyl cellulose modified keratin films," Journal of Textile Research, vol. 40, no. 6, 2019.

[18] M. Fatemah, Al-Douseri, Y. Q. Chen, and X.-C. Zhang, “THz wave sensing for petroleum industrial applications," International Journal of Infrared and Millimeter Waves, vol. 27, no. 4, pp. 481-503, 2006.

[19] T. D. Dorney, R. G. Baraniuk, and D. M. Mittleman, "Material parameter estimation with terahertz time-domain spectroscopy," Journal of the Optical Society of America A, vol. 18, no. 7, pp. 1562-1571, 2001.

[20] J. T. Kindt and C. A. Schmuttenmaer, "Far-infrared dielectric properties of polar liquids probed by femtosecond terahertz pulse spectroscopy," The Journal of Physical Chemistry, vol. 100, no. 24, pp. 10373-10379, 1996. 
[21] H. Son, D.-H. Choi, and G.-S. Park, "Improved thickness estimation of liquid water using Kramers-Kronig relations for determination of precise optical parameters in terahertz transmission spectroscopy," Optics Express, vol. 25, no. 4, pp. 4509-4518, 2017.

[22] Z. Q. Yang, Z. Y. Chai, and H. Y. Zhang, "Effect of electric field on water diffusion on kaolinite surface," Journal of China Coal Society, vol. 9, 2020. 